University of Nebraska - Lincoln

DigitalCommons@University of Nebraska - Lincoln

May 1990

\title{
Ordering of metal overlayers on metal substrates studied using atom beam scattering
}

\author{
G. Vidali \\ Syracuse University \\ C.W. Hutchings \\ Syracuse University \\ Peter A. Dowben \\ University of Nebraska-Lincoln, pdowben@unl.edu \\ M. Karimi \\ Alabama A\&M University, Normal \\ C. Moses \\ Utica College, Utica, New York \\ See next page for additional authors
}

Follow this and additional works at: https://digitalcommons.unl.edu/physicsdowben

Part of the Physics Commons

Vidali, G.; Hutchings, C.W.; Dowben, Peter A.; Karimi, M.; Moses, C.; and Foresti, M. , "Ordering of metal overlayers on metal substrates studied using atom beam scattering" (1990). Peter Dowben Publications. 136.

https://digitalcommons.unl.edu/physicsdowben/136

This Article is brought to you for free and open access by the Research Papers in Physics and Astronomy at DigitalCommons@University of Nebraska - Lincoln. It has been accepted for inclusion in Peter Dowben Publications by an authorized administrator of DigitalCommons@University of Nebraska - Lincoln. 


\section{Authors}

G. Vidali, C.W. Hutchings, Peter A. Dowben, M. Karimi, C. Moses, and M. Foresti 


\title{
Ordering of metal overlayers on metal substrates studied using atom beam scattering
}

\author{
G. Vidali, C.W. Hutchings, and P. A. Dowben \\ Physics Department, Syracuse University, Syracuse, New York 13244-1130 \\ M. Karimi \\ Physics Department, Alabama A\&M University, Nommal, Alabama 35762 \\ C. Moses \\ Physics Department, Utica College, Utica, New York 13502 \\ W. Foresti \\ Dipartimento di Fisica, Università di Campobasso, Campobasso, Italy
}

(Received 16 October 1989; accepted 30 October 1989)

\begin{abstract}
We will illustrate how atom beam scattering (ABS) can be used to study the growth and ordering of adsorption of $\mathrm{Hg}$ on $\mathrm{Cu}(001)$. At low coverage (less than $2 \%$ of a monolayer), we obtain that the scattering cross section of He with (adsorbed) Hg is about $50 \AA^{2}$. We find that mercury forms two stable phases on $\mathrm{Cu}(001)$ in the temperature range between 200 and $330 \mathrm{~K}, \mathrm{ac}(2 \times 2)$ and a high density $c(4 \times 4)$ phase. From ABS diffraction data, we can deduce the corrugation of the surface electron charge density of the mercury layer in the two ordered phases. From the analysis of our data, we obtain a Debye temperature for the $\mathrm{Hg}$ overlayer of about $115 \mathrm{~K}$. We will report preliminary calculations on the adsorption energy of $\mathrm{Hg}$ on $\mathrm{Cu}(001)$ and on the interaction of $\mathrm{He}$ with a $\mathrm{Hg}$ preplated $\mathrm{Cu}(001)$ surface.
\end{abstract}

\section{INTRODUCTION}

Improvements in characterizing and probing surfaces as well as an increased interest in the microscopic properties of surfaces and interfaces have spurred a great deal of research on the structural characteristics of ultrathin films on crystalline surfaces. ${ }^{1}$ In this work, we will illustrate how the combined use of two surface sensitive techniques, atom beam scattering (ABS), and low-energy electron diffraction (LEED), can be exploited to obtain detailed information on the growth and ordering of mercury on $\mathrm{Cu}(001)$.

We chose to study the $\mathrm{Hg} / \mathrm{Cu}(001)$ system for several reasons. First, there has been considerable interest lately in the electronic structure of mercury layers on metal surfaces. ${ }^{2}$ Particular attention was given to the issue of the metal-insulator transition. ${ }^{3}$ Second, the fact that the mercury layer could be easily deposited and removed made the $\mathrm{Hg} / \mathrm{Cu}$ system an ideal candidate for studies of growth and ordering of overlayers on metal substrates. Because of the large size of the $\mathrm{Hg}$ atom and the weak chemisorption of $\mathrm{Hg}$ on many metal surfaces, ${ }^{2.3}$ we expected to find that a system such as $\mathrm{Hg}$ on $\mathrm{Cu}(001)$ would show interesting phenomena as the result of a competition between adsorbate-adsorbate and adsorbate-substrate interactions. Finally, recent reports of ultraviolet photoelectron spectroscopy (UPS) studies of $\mathrm{Hg}$ on $\mathrm{Cu}(001)$ indicated a change in the electronic structure of $\mathrm{Hg}$, which could be related to the strain associated with the formation of an $\mathrm{Hg}$ layer with lattice constant $20 \%$ greater than the nearest neighbor distance in bulk $\mathrm{Hg}^{4}{ }^{4}$

As in a preliminary study to this system, ${ }^{5}$ LEED was used to obtain the phase diagram and for a rapid determination at the ordered structures of the overiayer. With ABS, out of the many possible interpretations of the LEED results, the overlayer structure could be uniquely identified. Additional information, such as the Debye temperature of the overlayer or the scattering cross section from isolated $\mathrm{Hg}$ atoms, have also been obtained by ABS, as will be described below.

\section{EXPERIMENTAL}

Our ABS apparatus is schematically shown in Fig. 1. It consists of a beam line, where the He beam is formed and collimated; a sample preparation chamber equipped with Ar ion sputtering gun, molecular-beam epitaxy (MBE), and evaporation sources for thin-film depositions, and a scattering chamber in which a reverse-view, four-grid LEED optics and a rotatable quadrupole mass spectrometer (QMS) are located. ${ }^{6}$ The He beam is formed by the expansion of up to 500 psi pressure of He gas through a $10 \mu$ nozzle into vacuum. The beam energy can be changed by cooling the nozzle with a Heli-Trans continuous flow cryostat. Beam energies of 21 and $63 \mathrm{meV}$ were used in this studiy.

The sample is mounted on a VG long-travel $(600 \mathrm{~mm})$ manipulator; the sample can then be moved from the sample preparation chamber into the scattering chamber. Operating pressures in the ultrahigh vacuum (UHV) chambers are in the mid $10^{-10}$ Torr range. The sample can be cooled down to $140 \mathrm{~K}$ and heated up to $1300 \mathrm{~K}$. The sample temperature was measured with a chromel-alumel thermocouple. The sample angles $(\theta$, polar angle made by the incoming beam and surface normal; $\phi$, azimuthal angle for rotation around sample normal) can be changed, as well the position of the manipulator axis.

The He beam is detected by a QMS (Balzers), which is housed in a differentially pumped enclosure. The detector is mounted on a doubly differentially pumped rotatable platform that can be rotated by $190^{\circ}$ around the manipulator axis by a stepping motor.

The $\mathrm{Cu}(001)$ sample is cleaned prior to each experiment by $A r$ ion sputtering $(1-2 \mathrm{kV}, 1 \mu \mathrm{A})$ and annealing up to $600^{\circ} \mathrm{C}$. Contamination can be monitored by a retarding field analyzer using Auger electron spectroscopy (AES). We determined that a better indicator of surface perfection was the measurement of the height and shape of the He specularly reflected beam. 


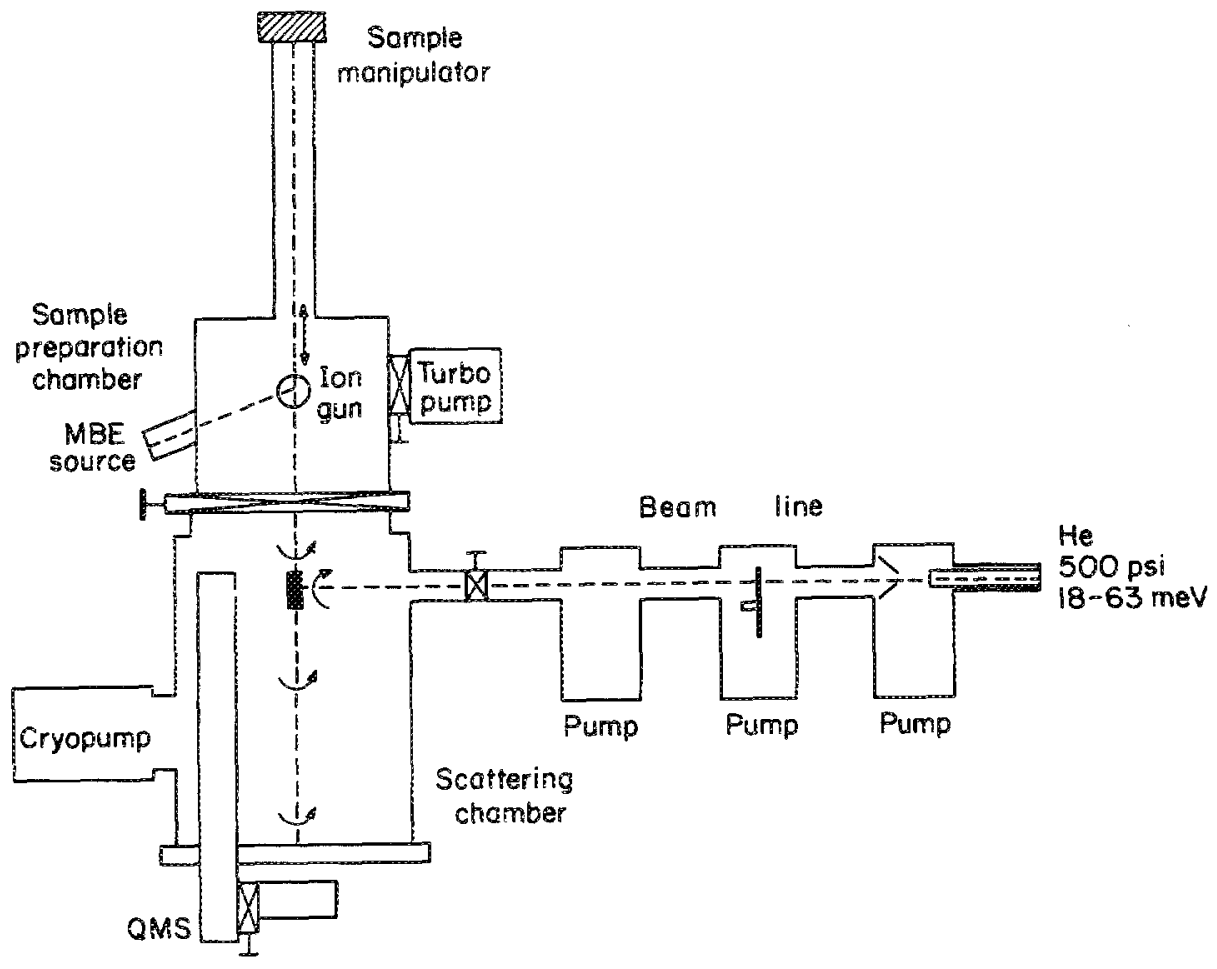

FIG. 1. Schernatic of the ABS apparatus.

Triple distilled mercury is admitted in the sample preparation chamber through a UHV leak valve. The pressure is measured by a nude ion gauge located in the scattering chamber. We found that mercury adsorbed only on a rather clean copper surface. Noticeable desorption occurs above room temperature, as measured by the attenuation of a diffraction beam of the overlayer with the reduction of the $\mathrm{Hg}$ ambient background pressure. Therefore, most of the adsorption experiments were done at temperatures less than $270 \mathrm{~K}$. Once a layer is formed, very little contamination is accumulated during a typical data acquisition interval $(1 / 2$ h); the surface, i.e., $\mathrm{Cu}(001)$ plus mercury layer, is periodically cleaned by flashing it to $200^{\circ} \mathrm{C}$. No trace of mercury is left on the surface and no amalgamation or alloying has been observed with UPS. ${ }^{4}$

\section{RESULTS}

In Fig. 2, we show the attenuation of the He specular intensity as a function of time, while mercury is admitted into the chamber at a pressure (uncorrected for ion gauge $\mathrm{Hg}$ cross section) of $3 \times 10^{-9}$ Torr. The coverage range explored in the experiment reported in Fig. 2 is from 0 to about $1.5 \%$ of a monolayer [here coverage is indicated as $\theta=\mathrm{Hg}$ atoms/Cu atoms on the (001) surface]. The surface temperature is $245 \mathrm{~K}$. As can be seen, the initial attenuation of the reflected He beam is linear in the mercury exposure. In Fig. 3, we show a complete adsorption curve; after the initial drop, the intensity of the specularly reflected He beam recovers. There is a kink in the curve at about 8.4 Langmuir (L) and the signal peaks at $9.8 \mathrm{~L}$. Here the $\mathrm{Hg}$ pressure is $1 \times 10^{-8}$ Torr and the surface temperature is $245 \mathrm{~K}$. LEED and ABS angular scans show that, at the coverages corresponding to the position of this kink, a $c(2 \times 2)$ structure with lattice parameter $a=3.6 \AA$ is formed on $\mathrm{Cu}(001)$ (the ideal coverage is $\theta=0.5$ ). Where the signal peaks in Fig. 3, a high density $c(4 \times 4)$ phase is formed (with coverage of 0.62 ). This structure corresponds to a square $\mathrm{Hg}$ lattice with next neighbor distance of $3.22 \AA$ rotated by $18.4^{\circ}$ from the $\langle 110\rangle$ direction of the $\mathrm{Cu}$ lattice. ${ }^{5}$

He diffraction scans for these two structures are reported in Fig. 4. These scans were taken at the position of the kink and peak as discussed above. The overlayer for each coverage studied was annealed to about $270 \mathrm{~K}$ and then the surface temperature was lowered to about $150 \mathrm{~K}$ to have a more favorable Debye-Waller factor. Care was taken to avoid desorbing or backadsorbing mercury in the heating/cooling cycles, respectively.

The attenuation of the specular He peak from a mercury layer as a function of surface temperature is reported in Fig. 5. From this figure, a Debye temperature for the $\mathrm{Hg}$ layer can be inferred as illustrated in the next section.

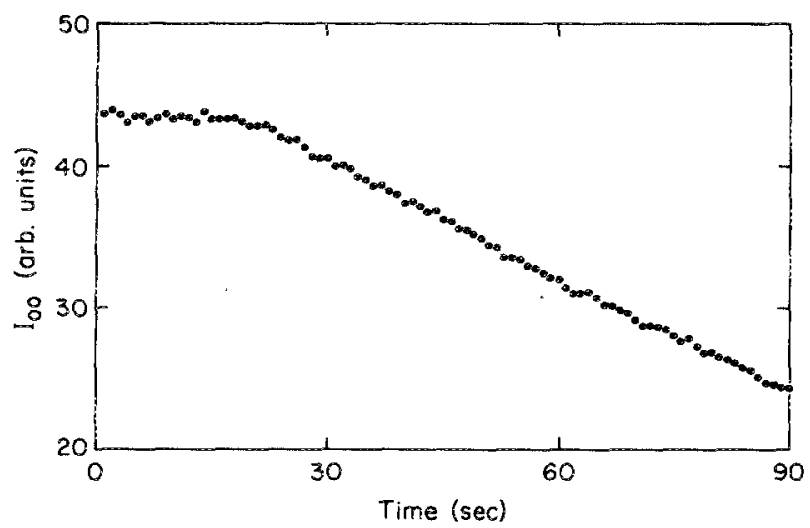

Fig. 2. Adsorption of $\mathrm{Hg}$ on $\mathrm{Cu}(001)$ measurcd by $\mathrm{He}$ specular scattering. $P_{\mathrm{Hg}}=3 \times 10^{\text {"}}$ Torr. $T_{s}=245 \mathrm{~K}$. The leak valve was opened at $t=20 \mathrm{~s}$. $\theta_{i}=70^{\circ}$. 


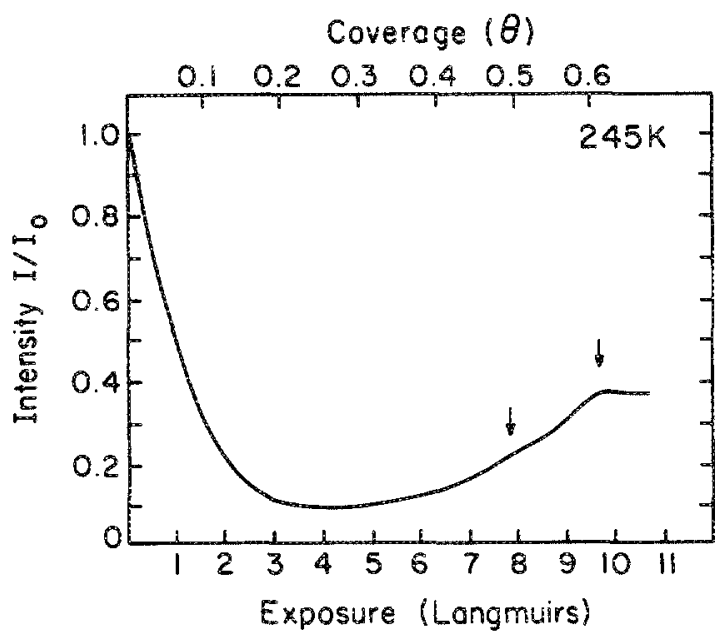

Fig. 3. Same as Fig. 2. $P_{\mathrm{Hg}}=1 \times 10^{-8}$ Torr. Left arrow corresponds to formation of $c(2 \times 2)$, right arrow to high density $c(4 \times 4)$.

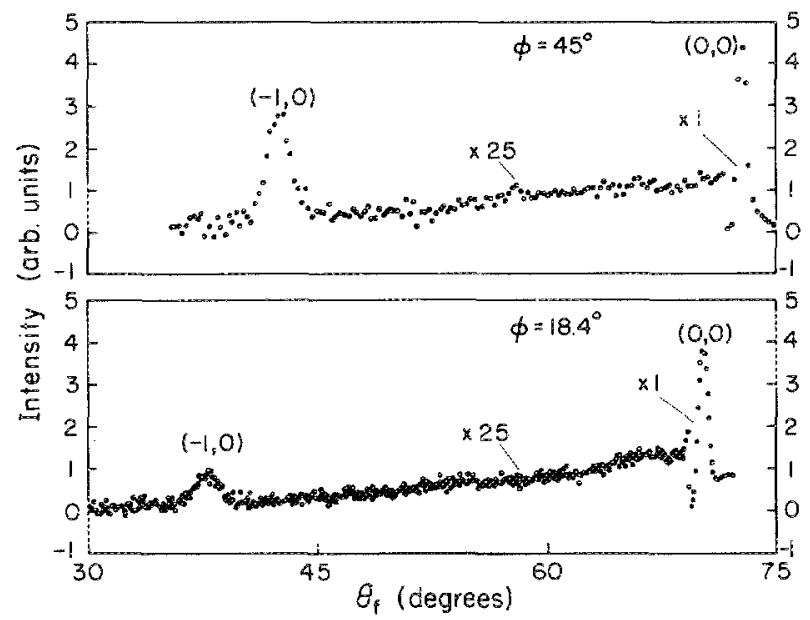

Fig. 4. He diffraction from a $c(2 \times 2)$ (top) and high density $c(4 \times 4)$ (bottom) $\mathrm{Hg}$ overlayer on $\mathrm{Cu}(001)$. Diffraction beams are labeled with respect to the overlayer net. Angle $\phi$ is with respect to the $\langle 1 \overline{1} 0\rangle$ direction in the copper crysta!. $E_{1}=21 \mathrm{meV} ; \theta_{i}=71.25^{\circ}$ (top) and $70^{\circ}$ (bottom).

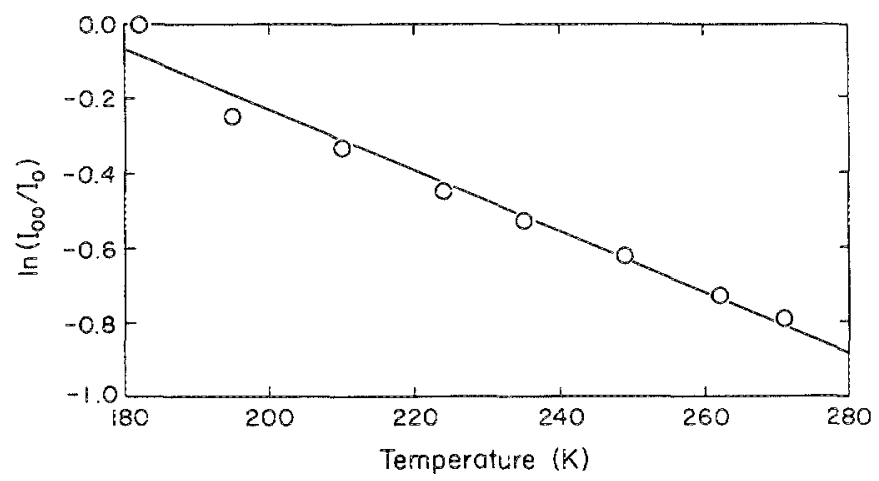

Fig. 5. Attenuation of the specular peak as a function of surface temperature for a high density $c(4 \times 4) \mathrm{Hg}$ overlayer on $\mathrm{Cu}(001) . E_{i} \ldots 63 \mathrm{meV} . \theta_{i}$ $=60^{\circ}$.

\section{DISCUSSION}

It has already been shown that He scattering is very sensitive to the presence of adsorbates at small concentrations. 7,8 It was found that the scattering cross section of He interacting with a single adsorbed atom or molecule is similar to the gas phase cross section for the same system. Intuitively, one can explain this by the face that in both cases it is the long range part of the interaction that leads to the scattering of $\mathrm{He}$ away from the specular direction.

Following $\operatorname{Ref} 7(b)$, we have

$$
I / I_{0}=(1-\theta)^{\alpha} \text {, }
$$

with $\alpha=\Sigma / A$, where $\Sigma$ is the He-Hg scattering cross section, $A$ is the area of the substrate unit cell and $I_{0}$ is the $\mathrm{He}$ specular intensity from the clean surface.

For small coverages, Eq. (1) yields a linear relationship between He intensity and coverage:

$$
I / I_{0}=(1-\theta)^{\alpha} \approx \exp (-\alpha \theta) \approx 1-\alpha \theta \text {. }
$$

From Fig. 3, we estimate that from the positions of the known ordered structures [i.e., $c(2 \times 2)$ and $c(4 \times 4)$ ] the sticking coefficient is approximately constant. We have also found that as the temperature is lowered, the exposures at which phase boundaries occur change little. ${ }^{5,9}$ In first approximation, we car take the sticking coefficient as unity. We then obtain from a linear fit to Fig. 2 that $\alpha \approx 8$ and therefore $\Sigma \approx 50 \AA^{2}$. The main uncertainty in this measurement is the error made in assuming that the sticking coefficient is unity. For comparison, the $\mathrm{He}-\mathrm{Pb}$ scattering cross section, when $\mathrm{Pb}$ is adsorbed on $\mathrm{Cu}(001)$, is about $80 \mathrm{~A}^{28}$ (the size of an isolated $\mathrm{Pb}$ is about $10 \%$ larger than $\mathrm{Hg}$ ).

We can address why the intensity of the specular peak increases again with increasing coverage, and why the original intensity of the He specularly scattered beam is not achieved upon completion of the overiayer as seen in Fig. 3. In the case of the scattering of $\mathrm{He}$ from $\mathrm{Xe}_{,}{ }^{10}$ it was seen that the attenuation rate of the He scattered intensity changed as $X e$ islands formed on the $P(111)$ surface, but the intensity was not observed to cease decreasing. We can explain our mercury on copper data in terms of the Debye-Waller factor. Using a conventional analysis of the Debye-Waller attenuation of a He beam scattering from a surface, ${ }^{6.11}$ we obtain

$$
\begin{aligned}
I / I_{0} & =\exp (-2 W) \\
& =\exp \left[-\frac{24 E_{i} T_{s}}{M k_{B} \theta_{D}^{2}}\left(\cos ^{2} \theta_{i}+D / E_{i}\right)\right],
\end{aligned}
$$

where $E_{i}$ is the incident beam energy, $T_{s}$ the surface temperature, $m$ and $M$ the mass of $\mathrm{He}$ and $\mathrm{Hg}$, respectively, $k_{B}$ the Boltzmann constant, $D$ is the $\mathrm{He}-\mathrm{Hg}$ plated $\mathrm{Cu}(001)$ potential well depth, and $\theta_{D}$ is the Debye temperature.

From an analysis of data in Fig. 5 we obtain that $\theta_{D}=115$ $\mathrm{K}$, where for $D$ we used the value of $5.5 \mathrm{meV}$, as discussed later. Using similar experimental procedures, we obtained the Debye--Waller factor for $\mathrm{Cu}(001)$; this gives an apparent Debye temperature for $\mathrm{Cu}(001)$, using the formula above, of $320 \mathrm{~K}$, a value that is comparable to what has been previously reported. "In principle, a more sophisticated analysis is necessary to take into account the simultaneous interaction 
of $\mathrm{He}$ with more than one $\mathrm{Cu}$ atom. In the case of the $\mathrm{He}-\mathrm{Hg}$ overlayer system, the distance between $\mathrm{Hg}$ atoms is much larger $(40 \%)$ and an interaction of a He atom with only one $\mathrm{Hg}$ atom is more likely.

The difference in the Debye-Waller factor for $\mathrm{He}$ $\mathrm{Cu}(001)$ and $\mathrm{He}-\mathrm{Hg} c(2 \times 2)$ is not as large as the one observed between $\mathrm{He}-\mathrm{Pt}(111)$ and $\mathrm{He}-\mathrm{Xe}$ on $\mathrm{Pt}(111) .^{10} \mathrm{We}$ note that in fact, Xe physisorbs on most surfaces, while $\mathrm{Hg}$ is weakly chemisorbed, as illustrated later.

We can now explain the source of differences in the signal for the $\mathrm{Hg}$ overlayer on $\mathrm{Cu}(001)$ as compared with the full intensity for $\mathrm{He}$ on $\mathrm{Cu}$; if we divide the $\mathrm{He}-\mathrm{Cu}$ intensity by the $\mathrm{He}-\mathrm{Hg}$ one, we obtain 0.37 [ here we took the intensity of the $c(4 \times 4)$ structure J. The ratio of the measured DebyeWaller factors gives 0.52 . The remaining discrepancy must be attributed to the fact that part of the He scatters in diffraction channels in the case of the mercury overlayer, while almost all the elastic intensity is channeled into the specular peak in the case of $\mathrm{He}-\mathrm{Cu}(001)$. Furthermore, the specular peak increases slightly upon annealing of the overlayer. We note that all the information obtained from figures such as Fig. 3 relates to the same area on the crystal. In such a case, the same defects and imperfection are monitored during the adsorption process.

It is of interest to know the "corrugation" of the surface, i.e., the variation of the electron surface charge distribution sampled by the He atom. This information is used in a variety of atom/molecule-surface processes, such as the infuence of corrugation on the change of rotational states of molecules on surfaces, ${ }^{12}$ on diffusion (hopping) coefficients, ${ }^{13}$ and on the determination of binding sites and defect distributions, ${ }^{14}$ to name a few.

To obtain an estimate of the amplitude of the He-surface potential modulation across the surface, which was demonstrated to reflect the modulation of the surface electron charge density, ${ }^{15}$ we used the scattering formalism of the hard wall model in the eikonal approximation. ${ }^{16,17}$ While we await a more complete set of data to perform the more sophisticated close coupling calculations, the method employed here should yield a good preliminary estimate on the surface corrugation, which could be compared with previous estimates for other systems.

The hard wall is represented by the function

$$
Z(z)=1 / 2 Z_{0}[\cos (2 \pi x / a)+\cos (2 \pi y / a)],
$$

where $a$ is the lattice spacing of the overlayer, while the probability of scattering in the channel $(m, n)$, where $(m, n)$ are integers appearing in the expression for the surface lattice vectors of the overlayer: $G=2 \pi / a(m, n)$, is given by

$$
P_{m, n}=\cos \theta_{g} / \cos \theta_{i}\left|A_{G}\right|^{2}
$$

with $\theta_{G}$ the diffracted angie and

$$
A_{G}=-\chi^{(\cdot i)^{i m \mid}: \mid n} J_{|m|}\left(c^{\prime}\right) J_{|n|}\left(c^{\prime}\right),
$$

where $\chi$ is a kinematical factor discussed in Ref. 14 and $J_{|m|}$ and $J_{n \mid}$ are Bessel functions of order $m$ and $n$ and $c^{\prime}$ is the momentum transfer corrected for the change in the particle momentum when it enters the well multiplied by $Z_{0}{ }^{16,17}$ This formalism should hold if the corrugation is not too large, i.e.,

$$
\frac{1}{2} Z_{0}<0.14 a,
$$

where $a$ is the lattice constant of a one-dimensional sinusoidal wall. ${ }^{4}$

From inspection of Fig. 4 and our calculations, we find this condition to be satisfed. For the $c(2 \times 2)$ phase, we find $\frac{1}{2} Z_{0}=0.08 \AA$, while for the high density $c(4 \times 4)$ phase we have $\frac{1}{2} Z_{0} \approx 0.03 \AA$.

These numbers can be compared with $0.3 \AA$ for He$\mathrm{LiF}(001),{ }^{18}$ i.e., a system with a large corrugation, $0.02 \AA$ for He-graphite (a smooth substrate) ${ }^{19}$ and less than 0.001 $\AA$ for $\mathrm{He}-\mathrm{Ag}(111),{ }^{20}$ the latter one is a typical one of close or nearly close pack surfaces of metals. For $\mathrm{Cu}(001)$, no $\mathrm{He}$ diffraction has been observed."

We have done a preliminary calculation of the $\mathrm{He}-\mathrm{Hg}$ overlayer on $\mathrm{Cu}(001)$ interaction potential. $\mathrm{Hg}$ atoms, in the $c(2 \times 2)$ configuration, were put on a $\mathrm{Cu}(001)$ surface; the distance of the $\mathrm{Hg}$ layer from the $\mathrm{Cu}$ surface (i.e., the distance between the planes of the nuclei) was found by calculating the equilibrium distance for a mercury atom interacting with the $\mathrm{Cu}(001)$ surface. We used Lennard-Jones $\mathrm{Hg}-\mathrm{Hg}^{21}$ and $\mathrm{Cu}-\mathrm{Cu}^{22}$ potentials and combination rules to obtain the $\mathrm{Hg}-\mathrm{Cu}$ potential. The $\mathrm{Hg}-\mathrm{Cu}$ layer distance was found to be $2.48 \AA$, while the binding energy for $\mathrm{Hg}$ on $\mathrm{Cu}(001) 0.52 \mathrm{eV}$ was in good agreement with the experimental value deduced from the isosteric heat at low coverage ( 0.05 of a monolayer) $0.48 \mathrm{eV}$.' The isosteric heats as a function of $\mathrm{Hg}$ coverage [up to the completion of the $c(2 \times 2)$ phase] were obtained from adsorption and desorption isobars where the coverages were determined by ABS. To obtain the He- $\mathrm{Hg}$ overlayer potential, we followed the procedure in Ref. 23 and used the He- $\mathrm{Hg}$ potential as determined by gas phase experiments. ${ }^{24}$ The resulting potential is shown in Fig. 6. The binding energy for $\mathrm{He}$ on the $\mathrm{Hg}$ layer is $5.5 \mathrm{meV}$. The calculations here reported do not have any adjustable parameter. We also obtained that the most favorable binding site for $\mathrm{He}$ on the $c(2 \times 2) \mathrm{Hg}-\mathrm{Cu}(001)$ surface is the four hollow one. The corrugation, i.e., the variation in the perpendicular direction of a isoenergy potential curve, is $Z_{0}=0.3 \AA$ at $18 \mathrm{meV}$ of energy, which is considerably higher than the hard wall estimate obtained above. We attribute this discrepancy chiefly to the inadequacy of the LennardJones model to describe the corrugation. Further work with more realistic potentials is under way.

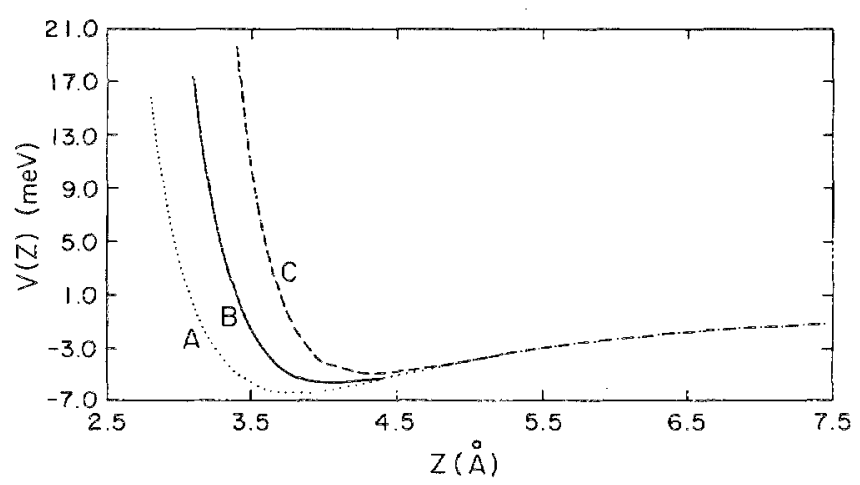

FIG. 6. $\mathrm{He}-c(2 \times 2) \mathrm{Hg} / \mathrm{Cu}(001)$ interaction potential. $\mathrm{C}$ is the potential calculated on top of an $\mathrm{Hg}$ atom; $B$ is on the bridge site, and $\mathrm{A}$ is on the fouffold hollow site. 


\section{CONCLUSIONS}

We have shown how ABS (and LEED) can be used to obtain very detailed information on the early stages of growth and ordering of a metal layer on a metal substrate. We applied our technique to the case of mercury overlayers on $\mathrm{Cu}(001)$. We found that at coverages less than $2 \%$ of a monolayer, the $\mathrm{He}-\mathrm{Hg}$ cross section is about $50 \AA^{2}$, signifcantly bigger than the hard sphere cross section (about 8 $\AA^{2}$ ). Two stable phases have been identified: a $c(2 \times 2)$, with nominal coverage of 0.5 , and a high density $c(4 \times 4)$, with coverage of 0.62 . An analysis of He diffraction intensities gives a corrugation parameter $Z_{0}$ of 0.16 and $0.06 \AA$ for the $c(2 \times 2)$ and $c(4 \times 4)$, respectively. We have also shown that He-monitored adsorption curves, in which the specular intensity of He is monitored as a function of overlayer coverage at a fixed surface temperature, can be very useful to obtain qualitative and quantitative information on the process of growth and ordering of the overlayer.

We are presertly working on obtaining information on phase transitions within the $\mathrm{Hg}$ overlayer using ABS.

\section{ACKNOWLEDGMENTS}

We would like to thank several collaborators who contributed to this work: W. Li, J. Havranek, and Y. J. Kime. Support for this work was provided by NSF DMR Grant No. 880250 . G. V. would like to thank the Sloan Foundation for supporting this work through a Fellowship. We acknowledge the support of the Alabama Supercomputer Network for Cray time and particularly Dr. D. S. Retallack for technical help.

'E. Bauer, in Structure and Dynamics of Surfaces II, edited by W. Schommers and P. von Blanckenhagen (Springer, Berlin, 1987), Vol. 43, p. 115 and references cited therein; Metallic Multilayers and Epitaxy edited by
M. Hong, D. U. Gubser, and S. A. Wolf (Metallurgical Society, Warrendale, 1988).

${ }^{2}$ P. A. Dowben, S. Varma, Y. J. Kime, D. R. Mueller, and M. Onellion, Z. Phys. B 73, 247 (1988).

${ }^{3}$ M. K. Sinh and R. G. Jones, Chem. Phys. Lett. 155, 463 (1989).

${ }^{4}$ M. Onellion, Y. J. Kime, P. A. Dowben, and N. Tache, J. Phys. C 20, L.633 (1987); P. A. Dowben, Y. J. Kime, D. LaGraffe, and M. Onellion, Surf. Interface Anal (to be published).

${ }^{5}$ A preliminary account of this work and information on the phase diagram appears in: C. W. Hutehings, P. A. Dowben, Y. J. Kime, W. Li, M. Karimi, C. Moses and G. Vidali, Mater. Sci. Soc. Symp. Proc. (to be published).

${ }^{6}$ G. Vidali and C. W. Hutchings, Phys. Rev. B 37, 10374 (1988).

${ }^{7}$ B. Poelsema, I. K. Verheij, and G. Comsa, Phys. Rev. Leit. 49, 1731 (1982); G. Comsa and B. Poelsema, Appl. Phys A 38, 153 (1985).

${ }^{2}$ A. Sanchez, J. Ibanez, R. Miranda, and S. Ferrer, J. Appl. Phys. 61,1239 (1987).

${ }^{9}$ P. A. Dowben, Y. J. Kime, C. W. Hutchings, W. Li, and G. Vidali, Surf. Sci. (in press).

${ }^{10}$ B. Poelsema, L. K. Verheij, and G. Comsa, Surf. Sci. 152/153, 851 (1985).

${ }^{17}$ J. Lapujoulade, J. Perreau, and A. Kara, Surf. Sci. 129, 59 (1983).

${ }^{12}$ J. A. Barker and D. J. Auerbach, Surf. Sci. Rep. 4, 1 (1985).

${ }^{13}$ M. Karimi and G. Vidali, in Diffusion at Interfaces: Microscopic Concepts, Springer Serics in Surface Science, Vol. 12 (Springer, New York, 1988), p. 43.

${ }^{14}$ T. Engel and K. H. Rieder, in Structural Studies of Surfaces with Atomic and Molecular Beam Diffraction, Springer Tracts in Modern Physics, Vol. 91 (Springer, Berlin, 1981 ); W. A. Schlup and K. H. Rieder, Phys. Rev. Lett. 56, 73 (1986).

${ }^{15}$ N. Esbjerg and J. K. Norskov, Phys. Rev. Lett. 45, 807 (1980).

${ }^{16}$ U. Garibaldi, A. C. Levi, R. Spadacini, and G. E. Tommei, Surf. Sci. 48, 649 (1975).

${ }^{17}$ V. Celli and D. Evans, in Dynamics of Gar-Surface Interaction, edited by G. Benedek and U. Valbusa, Springer Series in Chemical Physics, Vol. 21 (Springer, New York, 1982), p. 2.

${ }^{18}$ G. Boato, P. Cantini, and L. Mattera, Surf. \$ci. 55, 141 (1976).

${ }^{19}$ G. Boato, P. Cantini, and R. Tatarek, J. Phys. F 6, 6237 (1976).

${ }^{20}$ G. Boato, P. Cantini, and R. Tatarek, Phy. Rev. Lett. 69,887 (1978).

${ }^{21} \mathrm{~J}$. Worster and N. H. March, Solid State Commun. 2, 245 (1964).

${ }^{22}$ S. M. Foiles, Phys. Rev. B 32, 3409 (1985).

${ }^{23}$ S. Chung, N. Holter, and W. M. Cole, Surf. Sci. 165, 466 (1986); Phys. Rev. B 31, 6600 (1985).

${ }^{24}$ M.-C. Duval, C. Jouvet, and B. Soep, Chem. Phys. Lett. 119, 317 (1985). 